\title{
ESTRATO DE REGENERAÇÃO NATURAL DE UM TRECHO DE FLORESTA ESTACIONAL SEMIDECIDUAL, VIÇOSA, MG ${ }^{1}$
}

\author{
Brena Karina Siqueira Franco², Sebastião Venâncio Martins³, Patrícia Carneiro Lobo Faria ${ }^{4}$, Guido \\ Assunção Ribeiro ${ }^{3}$ e Aurino Miranda $\mathrm{Neto}^{5}$
}

\begin{abstract}
RESUMO - Este estudo foi realizado na Mata da Praça de Esportes, situada no Campus da UFV, em Viçosa, MG, com 10,65 ha em regeneração há 75 anos. Foram estabelecidos quatro transectos paralelos de 3 x 50 m, espaçados por $3 \mathrm{~m}$ e subdivididos em 10 parcelas de 3 x 5 m cada. A amostragem do estrato de regeneração natural incluiu todos os indivíduos com altura $\geq 0,3$ m e com DAP $<5 \mathrm{~cm}$. Calcularam-se os parâmetros fitossociológicos, e realizou-se a classificação quanto à classe sucessional e à síndrome de dispersão. Comparou-se a composição florística entre a área estudada e diversos levantamentos florísticos de regeneração na região de Viçosa, MG, por meio de análise de agrupamento. Foram obtidas 30 famílias e 84 espécies. As espécies mais importantes foram Coffea arabica, Siparuna guianensis, Psychotria sessilis e Erythroxylum pelleterianum. Houve predomínio da classe sucessional secundária tardia (49,3\% dos indivíduos) e da síndrome de dispersão zoocórica (91,6\% dos indivíduos). Não houve similaridade florística entre o trecho de floresta estudada e os demais fragmentos florestais da região. A predominância da síndrome de dispersão zoocórica no estrato de regeneração natural é fator importante para a continuidade da manutenção de animais frugívoros na área. A riqueza florística e a abundância de espécies secundárias tardias são fortes indicativos de que o ambiente estudado está oferecendo condições ecológicas adequadas ao desenvolvimento dessas espécies, permitindo, assim, o avanço da sucessão secundária.
\end{abstract}

Palavras-chave: Sub-bosque; Similaridade florística; Fitossociologia.

\section{NATURAL REGENERATION LAYER OF A SEMIDECIDUOUS FOREST FRAGMENT IN VIÇOSA, MINAS GERAIS STATE, BRAZIL}

\begin{abstract}
The present study was carried out at Praça de Esportes Forest, located on the campus of the Universidade Federal de Viçosa, Viçosa, Minas Gerais State. The fragment consists of a 10.65 ha forest in regeneration for 75 years. Four parallel transects mesuring $3 \times 50 \mathrm{~m}$, spaced $3 \mathrm{~m}$, and divided into 10 plots of $3 \times 5 \mathrm{~m}$ each were set for analysis. Sampling of natural regeneration layer included all individuals with height $\geq 0.3$ $m$ and $\mathrm{DBH}<5 \mathrm{~cm}$. We calculated the phytosociological parameters and classified the fragments into successional class and dispersion syndrome. The floristic composition was compared with several studies of regeneration in Viçosa, through cluster analysis. After analysis, we observed the occurrence of 30 families and 84 species, which the most important were Coffea arabica, Siparuna guianensis, Psychotria sessilis and Erythroxylum pelleterianum. The late secondary successional class was the most representative (49.3\% of individuals), and $91.6 \%$ of individuals presented dispersion syndrome zoochorous. No floristic similarity was observed between the studied area and other forest fragments of the region. The predominance of dispersion syndrome zoochorous in natural regeneration is an important factor to the continued maintenance of frugivorous in the area. The floristic richness and abundance of late secondary species are strong indications that the environment studied is offering ecological conditions suitable for the development of these species, thus allowing the advancement of secondary succession.
\end{abstract}

Keywords: Understory; Floristic similarity; Phytosociology.

\footnotetext{
${ }^{1}$ Recebido em 02.12.2012 aceito para publicação em 02.12.2013

${ }^{2}$ Escola Superior São Francisco de Assis (ESFA), Santa Teresa, ES. E-mail: <brenafranco@yahoo.com.br>.

${ }^{3}$ Departamento de Engenharia Florestal da Universidade Federal de Viçosa, UFV, Brasil. E-mail: <venancio@ufv.br>e <gribeiro@ufv.br>.

${ }^{4}$ Universidade Tecnológica Federal do Paraná, Brasil. E-mail: <patricialobo@utfpr.edu.br>.

${ }^{5}$ Programa de Pós-Graduação em Ciência Florestal, Universidade Federal de Viçosa, Departamento de Engenharia Florestal, Brasil. E-mail: <aur.neto@gmail.com>.
} 


\section{INTRODUÇÃO}

Na Região Sudeste do Brasil, o desenvolvimento econômico caracterizou-se inicialmente pela expansão da fronteira agrícola, ampliação da malha viária e, posteriormente, pelo aumento das áreas urbanas, atividades que, devido ao mau planejamento, exerceram e ainda exercem forte pressão sobre a cobertura florestal original. Assim, a fragmentação das Florestas Estacionais Semideciduais tem acarretado inúmeras alterações em suas características originais, como: diminuição da diversidade biológica, distúrbios do regime hidrológico de bacias hidrográficas, mudanças climáticas, degradação dos recursos naturais e deterioração da qualidade de vida (VIANA, 1990). A vegetação florestal da região denominada Zona da Mata de Minas Gerais, onde está inserido o Município de Viçosa, também sofreu esse processo de fragmentação (VALVERDE, 1959; MEIRA-NETO et al., 1997), resultando em inúmeras pequenas florestas secundárias em processo de regeneração natural, que foram abandonadas após o desmatamento.

A regeneração natural é definida como o processo pelo qual a floresta perturbada atinge características de floresta madura (KLEIN, 1980), por meio de processos sucessionais que ocorrem após distúrbios, como o corte e queima de árvores e arbustos, com posterior utilização do solo para atividades agropastoris; a abertura de clareiras naturais, causada pela queda de uma ou mais árvores do dossel; deslizamentos; atividades vulcânicas; e ataques de insetos (UHL et al., 1981; KAUFFMAN, 1991; COCHRANE; SCHULZE, 1999; MARTINS; RIBEIRO, 2002). Contudo, esse processo de reconstituição da comunidade florestal ocorre também na ausência desses distúrbios, por meio do crescimento dos indivíduos jovens de espécies arbustivo-arbóreas, que se desenvolvem sob a sombra do dossel das árvores até atingirem os estratos superiores da floresta, constituindo o estrato de regeneração natural.

Considerando que as florestas secundárias têm-se tornado muito comuns nas regiões tropicais (BROWN; LUGO, 1990), a realização de estudos sobre esses ecossistemas é de suma relevância para se conhecerem a estrutura, a diversidade e o seu funcionamento e, portanto, propor medidas de conservação e manejo dessas comunidades, assim como fornecer subsídios a práticas de restauração de ecossistemas degradados.
Dessa forma, este trabalho teve como objetivo caracterizar o estrato de regeneração natural de um trecho de Floresta Estacional Semidecidual, por meio da análise da sua composição florística.

\section{MATERIAL E MÉTODOS}

O estudo foi realizado em um trecho da Mata da Praça de Esportes, um fragmento florestal localizado no Campus da Universidade Federal de Viçosa, no Município de Viçosa, Minas Gerais (2045’S e 4251’W), cuja área compreende 10,65 ha. O clima da região, de acordo com a classificação de Köppen, é Cwb, com chuva mal distribuída ao longo do ano, verão chuvoso e inverno seco. O total pluviométrico anual médio é de $1.221 \mathrm{~mm}$ e a temperatura média, $19^{\circ} \mathrm{C}$ (VIANELLO, 1991). O relevo é acidentado, fazendo parte do chamado domínio do "Mar de Morros”. Quanto aos tipos de solo, predominam o Latossolo Vermelho-Amarelo no topo de morros; o Latossolo Câmbico nas encostas das elevações; e o Neossolo Flúvico associado aos Gleissolos no fundo dos vales (REZENDE, 1971; CORRÊA, 1984; RESENDE, 1986; EMBRAPA, 1999). A vegetação natural da região é classificada de acordo com o sistema fisionômico ecológico proposto por Veloso et al. (1991), como Floresta Estacional Semidecidual.

De acordo com Golfari (1975), até a década de 1920 a área foi utilizada para o cultivo do café (Coffea arabica L.). Segundo relatos de moradores da Vila Secundino, localizada no entorno do fragmento, alguns locais foram utilizados para plantio de eucalipto na década de 1980, onde ainda existem indivíduos remanescentes dessa espécie. Atribui-se a esse fragmento florestal cerca de 75 anos de idade.

Em um trecho com melhores condições de preservação, foram instalados quatro transectos paralelos de 3 x $50 \mathrm{~m}$, espaçados por $3 \mathrm{~m}$, localizados no interior da floresta, distantes $100 \mathrm{~m}$ da borda. Cada transecto foi subdividido em 10 parcelas de 3 x 5 m, compreendendo uma área amostral de $600 \mathrm{~m}^{2}$.

A amostragem do estrato de regeneração natural foi realizada no período de junho a dezembro de 2003, para a qual foram incluídos todos os indivíduos arbustivoarbóreos com altura igual ou superior a 0,30 m e com DAP (diâmetro a 1,30 m do solo) $<5,0 \mathrm{~cm}$.

A análise florística foi realizada com base no sistema filogenético proposto pelo APG III (2009). O material coletado foi prensado e herborizado no Herbário VIC 
da Universidade Federal de Viçosa (UFV). O reconhecimento das espécies foi realizado por meio de comparação com as coleções do Herbário VIC. Os nomes científicos e respectivos autores foram atualizados por meio do site www.mobot.org (Missouri Botanical Garden).

Os parâmetros fitossociológicos foram calculados por meio do programa FITOPAC 2.1 (SHEPHERD, 2010). Calcularam-se os parâmetros fitossociológicos (densidade, frequência, dominância, valor de importância), descritos por Mueller-Dombois e Ellenberg (1974); o índice de diversidade de Shannon-Wiener (H') (MAGURRAN, 1988); e a equabilidade (J') (PIELOU, 1975).

As espécies arbustivo-arbóreas identificadas no estudo foram classificadas em categorias sucessionais (pioneira, secundária inicial, secundária tardia, sem caracterização), de acordo com Gandolfi et al. (1995). Em cada espécie foi considerada, ainda, a sua síndrome de dispersão (zoocórica, anemocórica, autocórica ou sem caracterização), conforme van der Pijl (1982) e Barroso et al. (1999).

A composição florística arbustivo-arbórea do estrato de regeneração natural da Mata da Praça de Esportes foi comparada com outros fragmentos de Florestas Estacionais Semideciduais estudadas na região de Viçosa, MG, disponíveis na literatura, por meio da análise de agrupamento (Tabela 1). O banco de dados consistiu de uma matriz binária de presença e ausência de espécies, com exclusões de identificações em nível de família e gênero.
Procedeu-se, inicialmente, à conversão da matriz de dados binários em matriz de medidas de proximidade (similaridade ou dissimilaridade) entre os pares de unidades de observação (JOHNSON; WICHERN, 1988). Assim, a partir das composições florísticas, construiu-se uma matriz de índices de similaridade, e gerou-se um dendrograma. Para essa análise, empregou-se o índice de similaridade de Jaccard (MUELLER-DOMBOIS; ELLENBERG, 1974), segundo a fórmula (equação 1):

$$
\mathrm{IJac}=\mathrm{c} /(\mathrm{a}+\mathrm{b}+\mathrm{c})
$$

em que:

IJac = índice de similaridade de Jaccard; a = número de espécies exclusivas da área $\mathrm{A}$; b = número de espécies exclusivas da área B; e c = número de espécies comuns às duas áreas.

Para interpretar a similaridade florística, foi utilizado o método média de grupo UPGMA (Unweighted Pair Group Method with Arithmetic Mean). As análises foram efetuadas no programa FITOPAC 2.1 (SHEPHERD, 2010).

\section{RESULTADOS}

Do levantamento florístico foram obtidas 30 famílias, representadas por 61 gêneros e 84 espécies. Dessas espécies, 69 foram identificadas em nível de espécie, 12 em nível de gênero e três até o nível de família. Coffea arabica e Citrus limonum foram as espécies exóticas que estiveram presentes na amostragem (Tabela 2). As famílias de maior riqueza florística foram Rubiaceae (14 espécies), Fabaceae (11), Myrtaceae (8) e Meliaceae (6).

Tabela 1 - Informações sobre as áreas utilizadas na comparação florística entre os levantamentos florísticos em estrato de regeneração natural em fragmentos de Floresta Estacional Semidecidual (REG 1 a REG 7) e o estrato de regeneração natural de um trecho da Mata da Praça de Esportes, Viçosa, MG (MPE). Cod = código do local; e A = área de amostragem.

Table 1 - Information on the areas used for floristic comparison between the floristic surveys on natural regeneration layer in semideciduous forest fragments (REG 1 to REG 7) and natural regeneration layer of a stretch of the Praça de Esportes Forest, Viçosa, MG (MPE). Cod = site code; and A = sample area.

\begin{tabular}{ccccc}
\hline Cod & Local & Característica & A (ha) & Autor \\
\hline REG 1 & Mata do Paraíso & Floresta inicial & 0,02 & Silva Júnior et al., 2004 \\
REG 2 & Mata do Paraíso & Floresta madura & 0,02 & Silva Júnior et al., 2004 \\
REG 3 & Mata do Paraíso & Floresta inicial & 0,20 & Higuchi et al., 2006 \\
REG 4 & Mata da Pedreira & Floresta inicial & 0,10 & Marangon et al., 2008 \\
REG 5 & Mata do Paraíso & Sub-bosque eucalipto & 0,10 & Souza et al., 2007 \\
REG 6 & Mata do Paraíso & Clareira & 0,04 & Martins et al., 2008 \\
REG 7 & Mata do Paraíso & Sub-bosque & 0,18 & Martins et al., 2008 \\
MPE & Mata Praça de Esportes & Floresta inicial & 0,06 & Este estudo \\
\hline
\end{tabular}


Tabela 2 - Espécies amostradas no estrato de regeneração natural de um trecho da Mata da Praça de Esportes, Viçosa, MG, com as respectivas formas de vida, categorias sucessionais, síndromes de dispersão e parâmetros fitossociológicos: $\mathrm{FV}$ = Forma de vida; $\mathrm{Ab}=$ Arbusto; $\mathrm{Av}=$ Árvore; $\mathrm{CS}$ = Categoria sucessional; $\mathrm{SC}=$ Sem caracterização; $\mathrm{St}=$ Secundária tardia; Si = Secundária inicial; $\mathrm{P}$ = Pioneira; SD = Síndrome de dispersão; Z = Zoocórica; An = Anemocórica; $\mathrm{Au}=$ Autocórica; NI = Número de indivíduos; $\mathrm{DR}$ = Densidade relativa; DoR = Dominância relativa; FR = Frequência relativa; e VI = Valor de importância.

Table 2 - Species sampled in natural regeneration layer of a stretch of Praça de Esporte Forest, Viçosa, MG, with their life forms, successional class, dispersal syndromes and phytosociological parameters: FV = Life Form; $A b=B u s h$; $A v=$ Tree $; C S$ = successional class; $S C=$ No Characterization; $S t=$ Late Secondary; $i$ = Initial Secondary; $P=$ Pioneer $; S D=$ Dispersal Syndrome; $Z=$ zoochory; $A n=$ anemochory; $A u=$ autochory; $N I=N u m b e r$ of individuals; $D R=$ relative density; $D o R=$ relative dominance; $F R=$ relative frequency; and $V I=$ Value of importance.

\begin{tabular}{|c|c|c|c|c|c|c|c|c|}
\hline Espécie & $\mathrm{FV}$ & CS & $\mathrm{SD}$ & NI & $\mathrm{DR}(\%)$ & $\operatorname{DoR}(\%)$ & FR(\%) & $\mathrm{VI}(\%)$ \\
\hline Coffea arabica $\mathrm{L}$. & $\mathrm{Ab}$ & SC & $\mathrm{Z}$ & 651 & 29,34 & 8,82 & 4,16 & 14,11 \\
\hline Siparuna guianensis Aubl. & Av & St & $\mathrm{Z}$ & 224 & 10,09 & 11,03 & 6,46 & 9,19 \\
\hline Psychotria sessilis Vell. & Av & St & $\mathrm{Z}$ & 355 & 16,00 & 6,29 & 4,98 & 9,09 \\
\hline Erythroxylum pelleterianum A. St. Hil. & Av & $\mathrm{Si}$ & $\mathrm{Z}$ & 94 & 4,24 & 8,76 & 4,49 & 5,83 \\
\hline Coutarea hexandra (Jacq.) K. Schum. & Av & St & $\mathrm{Z}$ & 162 & 7,30 & 6,69 & 3,50 & 5,83 \\
\hline Tabernaemontana fuchsiaefolia A. DC. & Av & $\mathrm{P}$ & An & 5 & 0,23 & 9,49 & 0,38 & 3,36 \\
\hline Amaioua guianensis Aubl. & Av & $\mathrm{Si}$ & $\mathrm{Z}$ & 50 & 2,25 & 3,94 & 3,50 & 3,23 \\
\hline Pseudopiptadenia contorta (DC.) G.P. Lewis \& M.P. Lima & Av & St & An & 56 & 2,52 & 1,85 & 4,49 & 2,95 \\
\hline Copaifera langsdorffii Desf. & Av & St & $\mathrm{Z}$ & 34 & 1,53 & 3,20 & 4,00 & 2,91 \\
\hline Protium warmingianum Marchand & Av & St & $\mathrm{Z}$ & 58 & 2,44 & 1,47 & 3,83 & 2,58 \\
\hline Platypodium elegans Vogel & Av & $\mathrm{Si}$ & An & 24 & 1,08 & 2,77 & 2,85 & 2,23 \\
\hline Ottonia propinqua Kunt & $\mathrm{Ab}$ & St & $\mathrm{Z}$ & 62 & 2,79 & 0,44 & 3,01 & 2,08 \\
\hline Myrcia splendens (Sw.) DC. & Av & $\mathrm{Si}$ & $\mathrm{Z}$ & 27 & 1,22 & 1,99 & 3,01 & 2,07 \\
\hline Nectandra oppositifolia Ness & Av & $\mathrm{Si}$ & $\mathrm{Z}$ & 24 & 1,08 & 1,09 & 2,85 & 1,67 \\
\hline Trichilia catigua A. Juss. & Av & St & $\mathrm{Z}$ & 24 & 1,08 & 1,00 & 2,85 & 1,64 \\
\hline Sorocea bomplandii (Baill.) W.C. Burger. Lanj. e Wess. Boer & Av & St & $\mathrm{Z}$ & 17 & 0,77 & 2,00 & 2,02 & 1,59 \\
\hline Trichilia pallida Sw. & Av & $\mathrm{Si}$ & $\mathrm{Z}$ & 16 & 0,72 & 1,59 & 2,35 & 1,55 \\
\hline Mabea fistulifera Mart. & Av & $\mathrm{P}$ & $\mathrm{Z}$ & 23 & 1,04 & 1,18 & 2,35 & 1,52 \\
\hline Anadenanthera peregrina (L.) Speg. & Av & $\mathrm{Si}$ & An & 34 & 1,53 & 1,11 & 1,86 & 1,50 \\
\hline Cupania vernalis Cambess. & Av & $\mathrm{Si}$ & $\mathrm{Z}$ & 10 & 0,45 & 2,34 & 1,53 & 1,44 \\
\hline Luehea grandiflora Mart. \& Zucc. & Av & $\mathrm{Si}$ & An & 19 & 0,86 & 1,04 & 2,02 & 1,30 \\
\hline Syagrus romanzoffiana (Cham.) Glassman & Av & $\mathrm{Si}$ & $\mathrm{Z}$ & 14 & 0,63 & 1,59 & 1,53 & 1,25 \\
\hline Piptadenia gonoacantha (Mart.) J.F. Macbr. & Av & $\mathrm{Si}$ & An & 14 & 0,63 & 1,65 & 1,37 & 1,22 \\
\hline Psychotria bahiensis DC. & $\mathrm{Ab}$ & St & $\mathrm{Z}$ & 18 & 0,81 & 0,35 & 2,19 & 1,12 \\
\hline Casearia ulmifolia Vahl. ex. Vent. & Av & $\mathrm{Si}$ & $\mathrm{Z}$ & 13 & 0,59 & 1,05 & 1,53 & 1,06 \\
\hline Dalbergia nigra (Vell.) Allemao ex. Benth. & Av & St & An & 12 & 0,54 & 0,72 & 1,69 & 0,98 \\
\hline Alseis floribunda Schott & Av & St & $\mathrm{Z}$ & 1 & 0,05 & 2,18 & 0,21 & 0,81 \\
\hline Psychotria hastisepala Müll. Arg. & $\mathrm{Ab}$ & St & $\mathrm{Z}$ & 11 & 0,50 & 0,24 & 1,53 & 0,76 \\
\hline Psychotria brevicollis Müll. Arg. & $\mathrm{Ab}$ & St & $\mathrm{Z}$ & 10 & 0,45 & 0,21 & 1,37 & 0,68 \\
\hline Guatteria nigrescens Mart. & Av & $\mathrm{Si}$ & $\mathrm{Z}$ & 3 & 0,14 & 1,21 & 0,54 & 0,63 \\
\hline Machaerium nyctitans (Vell.) Benth. & Av & $\mathrm{Si}$ & An & 9 & 0,41 & 0,24 & 1,20 & 0,62 \\
\hline Plinia peruviana (Poir.) Govaerts & Av & St & $\mathrm{Z}$ & 7 & 0,32 & 0,28 & 1,20 & 0,60 \\
\hline Piper lindbergii C. DC. & $\mathrm{Ab}$ & St & $\mathrm{Z}$ & 12 & 0,54 & 0,21 & 1,04 & 0,60 \\
\hline Ocotea sp. & Av & SC & $\mathrm{Z}$ & 17 & 0,77 & 0,15 & 0,87 & 0,60 \\
\hline Cabralea canjerana (Vell.) Mart. & Av & $\mathrm{Si}$ & $\mathrm{Z}$ & 2 & 0,09 & 1,11 & 0,38 & 0,53 \\
\hline Apuleia leiocarpa (Vogel) J.F. Macbr. & Av & $\mathrm{Si}$ & $\mathrm{Au}$ & 1 & 0,05 & 1,25 & 0,21 & 0,50 \\
\hline Brunfelsia uniflora (Pohl.) D. Dom & $\mathrm{Ab}$ & SC & $\mathrm{Z}$ & 3 & 0,14 & 0,97 & 0,38 & 0,50 \\
\hline Trichilia lepidota Mart. & Av & St & $\mathrm{Z}$ & 1 & 0,05 & 1,20 & 0,21 & 0,49 \\
\hline Casearia decandra Jacq. & Av & St & $\mathrm{Z}$ & 2 & 0,09 & 0,94 & 0,37 & 0,47 \\
\hline Casearia arborea (Rich.) Urb. & Av & $\mathrm{Si}$ & $\mathrm{Z}$ & 4 & 0,18 & 0,63 & 0,53 & 0,45 \\
\hline Leandra niangaeformis Cogn. & $\mathrm{Ab}$ & SC & $\mathrm{Z}$ & 6 & 0,27 & 0,15 & 0,70 & 0,37 \\
\hline
\end{tabular}


Tabela 2 - Cont.

Table 2 - Cont.

\begin{tabular}{|c|c|c|c|c|c|c|c|c|}
\hline Espécie & FV & CS & $\mathrm{SD}$ & NI & $\mathrm{DR}(\%)$ & $\operatorname{DoR}(\%)$ & $\mathrm{FR}(\%)$ & $\mathrm{VI}(\%)$ \\
\hline Mollinedia schottiana (Spreng.) Perkins & Av & SC & $\mathrm{Z}$ & 6 & 0,27 & 0,14 & 0,70 & 0,37 \\
\hline Dictyoloma vandellianum A. Juss. & Av & $\mathrm{Si}$ & $\mathrm{Z}$ & 5 & 0,23 & 0,17 & 0,70 & 0,37 \\
\hline Piper mollicomum Kunth. & $\mathrm{Ab}$ & St & $\mathrm{Z}$ & 4 & 0,18 & 0,10 & 0,70 & 0,33 \\
\hline Myrtaceae 3 & Av & SC & $\mathrm{Z}$ & 4 & 0,18 & 0,09 & 0,70 & 0,32 \\
\hline Faramea multiflora A. Rich. & $\mathrm{Ab}$ & St & $\mathrm{Z}$ & 5 & 0,23 & 0,15 & 0,53 & 0,30 \\
\hline Cybistax antisyphilitica Mart. & Av & $\mathrm{Si}$ & An & 1 & 0,05 & 0,62 & 0,20 & 0,29 \\
\hline Ocotea odorifera (Vell.) Rohwer & Av & St & $\mathrm{Z}$ & 3 & 0,14 & 0,17 & 0,53 & 0,28 \\
\hline Machaerium brasiliense Vogel & Av & $\mathrm{Si}$ & An & 4 & 0,18 & 0,12 & 0,53 & 0,28 \\
\hline Guapira opposita (Vell.) Reitz & Av & St & $\mathrm{Z}$ & 3 & 0,14 & 0,13 & 0,53 & 0,27 \\
\hline Zanthoxylum rhoifolium Lam. & Av & $\mathrm{P}$ & $\mathrm{Z}$ & 3 & 0,14 & 0,09 & 0,53 & 0,25 \\
\hline Jacaranda macrantha Cham. & Av & $\mathrm{Si}$ & An & 2 & 0,09 & 0,21 & 0,37 & 0,22 \\
\hline Annona cacans Warm. & Av & $\mathrm{Si}$ & $\mathrm{Z}$ & 1 & 0,05 & 0,42 & 0,20 & 0,22 \\
\hline Vismia guianensis (Aubl.) Choisy & Av & $\mathrm{P}$ & $\mathrm{Z}$ & 1 & 0,05 & 0,42 & 0,20 & 0,22 \\
\hline Citrus limonum Risso & Av & SC & $\mathrm{Z}$ & 3 & 0,14 & 0,16 & 0,37 & 0,22 \\
\hline Eugenia sp. & Av & SC & $\mathrm{Z}$ & 2 & 0,09 & 0,18 & 0,37 & 0,21 \\
\hline Eugenia florida DC. & Av & SC & $\mathrm{Z}$ & 2 & 0,09 & 0,16 & 0,37 & 0,21 \\
\hline Psychotria sp. 1 & $\mathrm{Ab}$ & St & $\mathrm{Z}$ & 3 & 0,14 & 0,11 & 0,37 & 0,21 \\
\hline Miconia sp. & $\mathrm{Ab}$ & SC & $\mathrm{Z}$ & 3 & 0,14 & 0,09 & 0,37 & 0,20 \\
\hline Psychotria nuda (Cham. \& Schtdl.) Wawra & $\mathrm{Ab}$ & St & $\mathrm{Z}$ & 2 & 0,09 & 0,13 & 0,37 & 0,20 \\
\hline Myrsine ferruginea (Ruiz \& Pav.) Spreng. & Av & $\mathrm{Si}$ & $\mathrm{Z}$ & 2 & 0,09 & 0,13 & 0,37 & 0,20 \\
\hline Matayba elaegnoides Radlk. & Av & $\mathrm{Si}$ & $\mathrm{Z}$ & 2 & 0,09 & 0,12 & 0,37 & 0,19 \\
\hline Licania sp. & Av & SC & $\mathrm{Z}$ & 2 & 0,09 & 0,11 & 0,37 & 0,19 \\
\hline Andira sp. & Av & St & $\mathrm{SC}$ & 2 & 0,09 & 0,11 & 0,37 & 0,19 \\
\hline Stryphnodendron sp. & Av & $\mathrm{P}$ & $\mathrm{Au}$ & 2 & 0,09 & 0,10 & 0,37 & 0,19 \\
\hline Nectandra sp. & Av & SC & $\mathrm{Z}$ & 2 & 0,09 & 0,08 & 0,37 & 0,18 \\
\hline Zanthoxylum riedelianum Engl. & Av & $\mathrm{Si}$ & $\mathrm{Z}$ & 1 & 0,05 & 0,22 & 0,20 & 0,16 \\
\hline Myrciaria sp. & Av & SC & $\mathrm{Z}$ & 1 & 0,05 & 0,17 & 0,20 & 0,14 \\
\hline Guarea pendula R.da Silva Ramalho, A.L. Pinheiro \& T.D. Penn & Av & St & $\mathrm{Z}$ & 2 & 0,09 & 0,12 & 0,20 & 0,14 \\
\hline Palicourea longepedunculata Gardner & $\mathrm{Ab}$ & St & $\mathrm{Z}$ & 3 & 0,14 & 0,07 & 0,20 & 0,14 \\
\hline Piptocarpha macropoda (DC.) Baker & Av & $\mathrm{P}$ & An & 1 & 0,05 & 0,16 & 0,20 & 0,14 \\
\hline Miconia pauciflora Triana & $\mathrm{Ab}$ & SC & $\mathrm{Z}$ & 1 & 0,05 & 0,14 & 0,20 & 0,13 \\
\hline Psychotria sp. 2 & $\mathrm{Ab}$ & St & $\mathrm{Z}$ & 1 & 0,05 & 0,10 & 0,20 & 0,12 \\
\hline Brosimum guianense (Aubl.) Huber & Av & $\mathrm{Si}$ & $\mathrm{Z}$ & 1 & 0,05 & 0,07 & 0,20 & 0,11 \\
\hline Lacistema pubescens Mart. & Av & $\mathrm{Si}$ & $\mathrm{Z}$ & 1 & 0,05 & 0,06 & 0,20 & 0,10 \\
\hline Allophylus sericeus (Cambess.) Radlk. & Av & $\mathrm{Si}$ & $\mathrm{Z}$ & 1 & 0,05 & 0,06 & 0,20 & 0,10 \\
\hline Sparattosperma leucanthum (Vell.) K. Schum. & Av & $\mathrm{Si}$ & An & 1 & 0,05 & 0,05 & 0,20 & 0,10 \\
\hline Ilex sp. & Av & SC & $\mathrm{Z}$ & 1 & 0,05 & 0,02 & 0,20 & 0,09 \\
\hline Myrtaceae 1 & Av & SC & $\mathrm{Z}$ & 1 & 0,05 & 0,01 & 0,20 & 0,09 \\
\hline Myrtaceae 2 & Av & SC & $\mathrm{Z}$ & 1 & 0,05 & 0,01 & 0,20 & 0,09 \\
\hline Endlicheria paniculata (Spreng.) J.F. Macbr. & Av & $\mathrm{Si}$ & $\mathrm{Z}$ & 1 & 0,05 & 0,01 & 0,20 & 0,09 \\
\hline Palicourea sp. & $\mathrm{Ab}$ & St & $\mathrm{Z}$ & 1 & 0,05 & 0,00 & 0,20 & 0,08 \\
\hline Miconia pusilliflora (DC.) Naudim & Av & SC & $\mathrm{Z}$ & 1 & 0,05 & 0,00 & 0,20 & 0,08 \\
\hline Leandra involucrata D.C. & $\mathrm{Ab}$ & SC & $\mathrm{Z}$ & 1 & 0,05 & 0,00 & 0,20 & 0,08 \\
\hline Total & & & & 2.219 & 100,00 & 100,00 & 100,00 & 100,00 \\
\hline
\end{tabular}

Foram amostrados 2.219 indivíduos, com densidade estimada em 36.983 indivíduos.ha-1. O número de plantas encontradas por unidade amostral variou de 17 a 143 . Os índices de diversidade de Shannon ( $\mathrm{H}^{\prime}$ ) foi de 2,811 e o de equabilidade (J), 0,631 .
Com relação ao VI (Valor de Importância) das famílias, destacaram-se Rubiaceae, Fabaceae, Monimiaceae e Erythroxylaceae, perfazendo 61,0\% do VI total. As cinco espécies com maiores VIs foram Coffea arabica, Siparuna guianensis, Psychotria sessilis e Erythroxylum pelleterianum (Tabela 2). 
Os maiores valores de frequência relativa (FR) foram para S. guianensis, $P$. sessilis, E. pelleterianum e Pseudopiptadenia contorta. Já os valores de dominância relativa (DR) foram mais elevados em $S$. guianensis, Tabernaemontana fuchsiaefolia, C. arabica e E. pelleterianum (Tabela 2).

Quanto à forma de vida, a arbórea foi predominante (64,1\% dos indivíduos), seguida da arbustiva (35,9\%). Os indivíduos arbustivo-arbóreos pertencentes à categoria sucessional das secundárias tardias representaram 49,3\% do total de indivíduos amostrados. Já a síndrome de dispersão com maior representatividade foi a zoocórica, com 91,6\% dos indivíduos arbustivoarbóreos amostrados (Tabela 3).

Das 84 espécies, 34,5\% são tardias, 35,7\% iniciais, $22,6 \%$ pioneiras, $7,2 \%$ sem características e, $81 \%$ zoocóricas, 15,5\% anemocóricas, 2,3\% autocóricas e 1,2\% sem características. Apesar de os indivíduos zoocóricos representarem 91,6\% do total, 50,4\% deles eram tardios, $13,4 \%$ iniciais, $34,8 \%$ pioneiras e $1,4 \%$ sem características; entretanto, quando analisadas quanto à composição de espécies, 38,3\% eram tardias, $39,4 \%$ iniciais, $4,4 \%$ pioneiras e $27,9 \%$ sem características.

O dendrograma comparando o estrato de regeneração natural da Mata da Praça de Esportes com outros fragmentos de Florestas Estacionais Semideciduais estudadas na região de Viçosa, MG, não evidenciou similaridade florística entre o trecho de floresta estudada e os demais fragmentos florestais (Figura 1). Apenas os fragmentos florestais REG 2, REG 6 e REG 7 apresentaram semelhança florística entre eles.

\section{DISCUSSÃO}

Alguns estudos realizados em Florestas Estacionais Semideciduais da Zona da Mata de Minas Gerais (PAULA et al., 2002; MEIRA-NETO; MARTINS, 2003; SILVA JÚNIOR et al., 2004; HIGUCHI et al., 2006) e do Estado de São Paulo(MARTINS; RODRIGUES, 2002; MARTINS et al., 2004) têm mostrado a importância das famílias em destaque neste trabalho, na composição florística desse ecossistema, com maior importância para a família Rubiaceae, em consequência do seu elevado número de espécies arbustivas características de sub-bosque.

De acordo com Tabarelli et al. (1994), desde os estádios iniciais da sucessão no domínio da Floresta Atlântica, Rubiaceae e Myrtaceae são famílias importantes no desenvolvimento da comunidade, principalmente do sub-bosque, enquanto Lauraceae e Meliaceae são indícios da passagem da floresta pioneira para um estádio sucessional mais avançado.

A família Fabaceae destaca-se em termos de riqueza de espécies e número de indivíduos em diversos estudos (VOLPATO, 1994; MARTINS; RODRIGUES, 2002; MEIRANETO; MARTINS, 2003; HIGUCHI et al., 2006). Segundo Campello (1998), essa família atende às necessidades de oferta contínua de nitrogênio e material orgânico de rápida decomposição e apresenta rápido estabelecimento da cobertura vegetal, além de promover mudanças microambientais que atuam como ativadoras e reguladoras dos recursos disponíveis, permitindo, assim, o estabelecimento de espécies mais exigentes no meio.

Silva Júnior et al. (2004), comparando duas áreas de Floresta Estacional Semidecidual, uma em estádio inicial de regeneração e outra em estádio avançado,

Tabela 3 - Categorias sucessionais e síndromes de dispersão dos indivíduos arbóreos e arbustivos amostrados no estrato de regeneração natural de um trecho da Mata da Praça de Esportes, Viçosa, MG. № = número de indivíduos; e \%I = percentual de indivíduos.

Table 3 - Successional class and dispersal syndromes of individual trees and shrubs sampled in natural regeneration layer of a stretch of Praça de Esportes Forest, Viçosa, MG. $N^{\circ} I=$ number of individuals; and \% I = percentage of individuals.

\begin{tabular}{|c|c|c|c|c|c|c|c|c|c|}
\hline \multicolumn{5}{|c|}{ Categorias sucessionais } & \multicolumn{5}{|c|}{ Síndromes de dispersão } \\
\hline & \multicolumn{2}{|c|}{ Árvores } & \multicolumn{2}{|c|}{ Arbustos } & \multirow[b]{2}{*}{ Classes } & \multicolumn{2}{|c|}{ Árvores } & \multicolumn{2}{|c|}{ Arbustos } \\
\hline Classes & $\mathrm{N}^{\circ} \mathrm{I}$ & $\% \mathrm{I}$ & $\mathrm{N}^{\circ} \mathrm{I}$ & $\% \mathrm{I}$ & & $\mathrm{N}^{\circ} \mathrm{I}$ & $\% \mathrm{I}$ & $\mathrm{N}^{\circ} \mathrm{I}$ & $\% \mathrm{I}$ \\
\hline Pioneira & 35 & 2,5 & 0 & 0,0 & Zoocoria & 1235 & 86,9 & 797 & 100,0 \\
\hline Secundária inicial & 381 & 26,8 & 0 & 0,0 & Anemocoria & 182 & 12,8 & 0 & 0,0 \\
\hline Secundária tardia & 963 & 67,7 & 132 & 16,6 & Autocoria & 3 & 0,2 & 0 & 0,0 \\
\hline S/ caracterização & 43 & 3,0 & 665 & 83,4 & S/ caracterização & 2 & 0,1 & 0 & 0,0 \\
\hline Total & 1422 & 100,0 & 797 & 100,0 & & 1422 & 100,0 & 797 & 100,0 \\
\hline
\end{tabular}

Revista Árvore, Viçosa-MG, v.38, n.1, p.31-40, 2014 


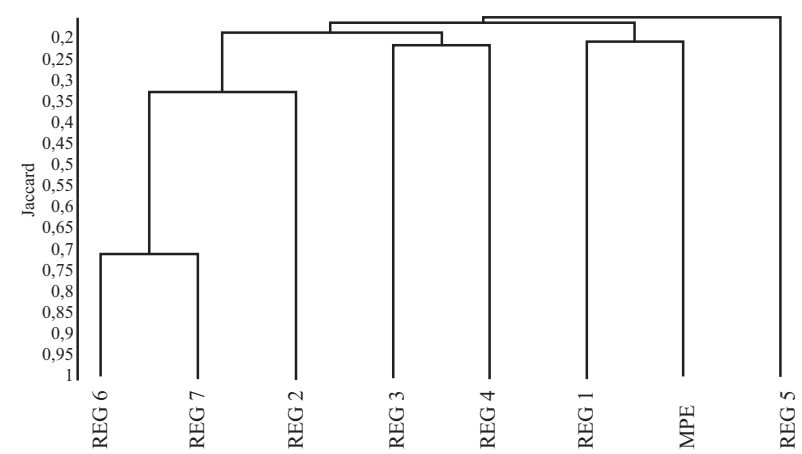

Figura 1 - Dendrograma da análise de agrupamento (UPGMA) utilizando o índice de similaridade de Jaccard para os levantamentos florísticos em estrato de regeneração natural em fragmentos de Floresta Estacional Semidecidual situados na região de Viçosa, MG (REG 1 a REG 6), e o estrato de regeneração natural de um trecho da Mata da Praça de Esportes, Viçosa, MG (MPE).

Figure 1 - Dendrogram of cluster analysis (UPGMA) using the Jaccard similarity index for the floristic surveys on natural regeneration layer in Semideciduous Forest fragments in the region of Viçosa, MG (REG REG 1 to 6), and natural regeneration layer of a stretch of the Praça de Esportes Forest, Viçosa, MG (MPE).

encontraram na primeira valores de diversidade e equabilidade de 1,91 e 0,45, respectivamente, e na segunda, valores de 3,15 (diversidade) e 0,79 (equabilidade). Os valores encontrados neste estudo indicaram que a Mata da Praça de Esportes situa-se entre o estádio inicial de regeneração e o estádio avançado encontrado por Silva Júnior et al. (2004).

A presença marcante de Coffea arabica tem sido constatada em estudos de fragmentos florestais do Sudeste brasileiro (SEVILHA et al., 2001; MARTINS; RODRIGUES, 2002; SILVA JÚNIOR et al., 2004). Essa presença reflete a influência de remanescentes (em forma de sementes, plântulas ou indivíduos jovens) dos antigos plantios de café da região e, de acordo com Bernacci e Leitão-Filho (1996), a atual disseminação por pássaros. A maior abundância da espécie neste estudo foi observada em ambientes sombreados da floresta, onde estava distribuída de forma agregada, formando populações dominantes em certas parcelas. Tal situação foi observada também por Sevilha et al. (2001) e Silva Júnior et al. (2004), em Florestas Estacionais Semideciduais de Viçosa, onde C. arabica estaria competindo com a regeneração natural nativa.
Entretanto, o estudo de Martins e Rodrigues (2002) demonstrou grande abundância de C. arabica em ambientes de clareira.

No Brasil, diversas variedades de C. arabica foram selecionadas para se desenvolverem a pleno sol, mas Grombone-Guaratini (1999) citaram que essa espécie tem sido caracterizada como climácica na dinâmica florestal, como modelo para a implantação de sistemas agroflorestais. Desse modo, sugere-se que C. arabica seja capaz de ocupar, de forma eficiente, os dois ambientes, sendo essa uma ameaça competitiva para as espécies nativas que compõem o estrato de regeneração natural dessas florestas.

Algumas das principais espécies (Psychotria sessilis, Siparuna guianensis e Erythroxylum pelleterianum) amostradas neste estudo também se destacaram em outros levantamentos da região de Viçosa (SILVAJÚNIOR et al., 2004; MEIRA-NETO; MARTINS, 2003). Essas espécies representaram $45,6 \%$ dos indivíduos dos grupos das secundárias iniciais e tardias, o que demonstra contribuição para o avanço da sucessão secundária desse ambiente.

Estudando várias clareiras numa Floresta Estacional Semidecidual do Estado de São Paulo, Martins e Rodrigues (2002) detectaram maior número de espécimes secundárias tardias no local de estudo, em função da predominância de pequenas clareiras, as quais favorecem o crescimento de espécies de sucessão mais avançada, em detrimento das pioneiras. As plântulas e, ou, os indivíduos jovens de espécies tardias são capazes de permanecer na mesma classe de tamanho por extensos períodos de tempo até que recursos como o espaço para o seu crescimento (LIEBERMAM, 1996) e a luz, decorrente da formação de pequenas clareiras na época de maior deciduidade da floresta, se tornem disponíveis.

A abundância dessas espécies na área de estudo indica que esse ambiente está oferecendo condições ecológicas adequadas ao seu desenvolvimento, sugerindo o avanço da sucessão nesse trecho de floresta.

As unidades de amostragem com menos indivíduos registrados estavam em aberturas do dossel da floresta e ocupadas por cipós e ervas invasores. Essas características podem ser o resultado da fragmentação da floresta, que expõe seu interior a situações como grande efeito de borda, já que não possui uma zona

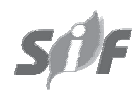

Revista Árvore, Viçosa-MG, v.38, n.1, p.31-40, 2014 
de amortecimento. Esse fato também está associado a perturbações do entorno, como ruídos constantes de veículos, que podem afugentar a fauna dispersora da área; a chegada de sementes de espécies invasoras; e a retirada de produtos florestais para fins medicinais, resultando na abertura de trilhas.

A elevada representatividade da dispersão zoocórica do trecho de floresta estudado corrobora estudo de Hartshorn (1980) sobre uma floresta onde 63\% das espécies eram dispersas por animais voadores (75\% por pássaros, $21 \%$ por morcegos e $4 \%$ por ambos), $8 \%$ pelo vento e $14 \%$ por animais terrestres. Nas florestas neotropicais, mais de $50 \%$ das plantas são dispersas por animais frugívoros (HOWE; SMALLWOOD, 1982), sendo geralmente aves e mamíferos os encarregados dessa disseminação (KUBITZKI; ZIBURSKI, 1994; MEDELÍN; GAONA, 1999). De acordo com Harper (1977), o tamanho da população de plantas é mais afetado pela dispersão de seus propágulos do que pelo seu número efetivo de indivíduos. Piña-Rodrigues (1990) afirmou que o potencial de estabelecimento de uma população vegetal em um hábitat é essencialmente controlado pelo fluxo de propágulos. Conforme essa autora, a dispersão zoocórica tem importante papel na manutenção das espécies de estádios sucessionais mais avançados e, indiretamente, na sua distribuição espacial e frequência das espécies da floresta.

Entre o estrato de regeneração natural da Mata da Praça de Esportes e os demais levantamentos florísticos na região de Viçosa, MG, houve dissimilaridade florística, uma vez que não alcançaram valor igual ou superior a 0,25, nível de semelhança florística considerável pelo índice de Jaccard (MUELLER-DUMBOIS; ELLENBERG, 1974). Apesar da proximidade das áreas, há diferenças no tamanho da área de amostragem e nas características do estrato de regeneração natural dos fragmentos florestais (sub-bosque de eucalipto, clareira, entorno de clareira, diferentes condições topográficas, diferentes estádios sucessionais). Tais fatos podem ter colaborado para a diminuição da similaridade entre os levantamentos.

Os levantamentos REG 2, REG 6 e REG 7 apresentaram similaridade, possivelmente pela mesma localização desses estudos, que foram realizados em um trecho da Mata do Paraíso, pertencente à Universidade Federal de Viçosa e preservado e livre de distúrbios antrópicos nas últimas cinco décadas, denominado floresta madura.

Revista Árvore, Viçosa-MG, v.38, n.1, p.31-40, 2014
Quando analisada em um mesmo tipo de ecossistema florestal, a similaridade florística entre áreas espacialmente próximas e dentro de uma mesma bacia hidrográfica éconsiderada elevada(RODRIGUES; NAVE, 2000). Porém, interferências diretas na similaridade florística podem ocorrer devido a fatores ambientais e espaciais entre áreas próximas (IVANAUSKAS et al., 2000; KUNZ et al., 2009), aumentando a dissimilaridade.

\section{CONCLUSÕES}

A predominância da síndrome de dispersão zoocórica no estrato de regeneração natural é fator importante para a continuidade da manutenção de animais frugívoros na área. A riqueza florística e a abundância de espécies secundárias tardias são indícios de que o tempo e o ambiente estudado oferecem condições ecológicas adequadas ao desenvolvimento dessas espécies, permitindo, assim, o avanço da sucessão secundária.

\section{REFERÊNCIAS}

APG III - Angiosperm Phylogeny Group III. An update of the Angiosperm Phylogeny Group classification for the orders and families of flowering plants. Botanical Journal of the Linnean Society, v.161, n.2, p.105-121, 2009.

BARRoso, G. M. et al. Frutos e sementes: morfologia aplicada à sistemática de dicotiledôneas. Viçosa, MG: Universidade Federal de Viçosa, 1999. 443p.

BERNACCI, L. C.; LEITÃO FILHO, H. F. Flora fanerogâmica da floresta da Fazenda São Vicente, Campinas, SP. Revista Brasileira de Botânica, v.19, n.1, p.149-164, 1996.

BROWN, S.; LUGO, A. E. Tropical secondary forests. Journal of Tropical Ecology, v.6, n.1, p.1-32, 1990.

CAMPELLO, E. F. C. Sucessão vegetal na recuperação de áreas degradadas. In: DIAS, L. E.; MELLO, J. W. V. (Ed.). Recuperação de áreas degradadas. Viçosa, MG: Universidade Federal de Viçosa, 1998. p.183-196.

COCHRANE, M.; SCHULZE, M. D. Fire as a recurrent event in tropical forests of the eastern Amazon: effects on forest structure, biomass, and species composition. Biotropica, v. 31, n.1, p.2-16, 1999. 
CORRÊA, G. F. Modelo de evolução e mineralogia da fração argila de solos do planalto de Viçosa: UFV. 1984. $187 \mathrm{f}$. Dissertação (Mestrado em Ciência Florestal) Universidade Federal de Viçosa, Viçosa, MG, 1984.

\section{EMPRESA BRASILEIRA DE PESQUISA}

AGROPECUÁRIA - EMBRAPA. Centro Nacional de Pesquisa de Solos. Sistema brasileiro de classificação de solos. Brasília: 1999. 412p.

GANDOLFI, S.; LEITÃO FILHO, H. F.; BEZERRA, C. L. F. Levantamento florístico e caráter sucessional das espécies arbustivo-arbóreas de uma floresta semidecídua no município de Guarulhos, SP. Revista Brasileira de Biologia, v.55, n.4, p.753-767, 1995.

GOLFARI, L. Zoneamento ecológico do Estado de Minas Gerais. Belo Horizonte: Centro de Pesquisa Florestal da Região do Cerrado, 1975. 65p.

GROMBONE-GUARATINI, M. T. Dinâmica de uma Floresta Estacional

Semidecidual: o banco, a chuva de sementes e o estrato de regeneração. 1999. 150f. Tese (Doutorado em Biologia Vegetal) - Universidade Estadual de Campinas, Campinas, 1999.

HARPER, J. L. Population biology of plants. London: Academic Press, 1977. 892p.

HARTSHORN, G. S. Neotropical forest dynamics. Biotropica, v.12 (supl.), p.23-30, 1980.

HIGUCHI, P. et al. Composição florística da regeneração natural de espécies arbóreas ao longo de oito anos em um fragmento de Floresta Estacional Semidecidual, em Viçosa, MG. Revista Árvore, v.30, n.6, p.893-904, 2006.

HOWE, H. F.; SMALLWOOD, J. Ecology of seed dispersal. Annual Review of Ecology and Systematics, v.13, p.201-228, 1982.

IVANAUSKAS, N. M.; MONTEIRO, R.; RODRIGUES, R. R. Similaridade florística entre áreas de Floresta Atlântica no estado de São Paulo. Brazilian Journal of Ecology, v.1, p.71-81, 2000.

JOHnsOn, R. A.; WICHERN, D. W. Applied multivariate statistical analysis. New Jersey: Prentice-Hall, 1988. 607p.
KAUFFMAN, J. B. Survival by sprouting following fire in tropical forest of the Eastern Amazon. Biotropica, v.23, p.219-224, 1991.

KLEIN, R. M. Ecologia da flora e vegetação do vale do Itajaí. Sellowia, v.32, n.1, p.165-389, 1980.

KUBITZKI, K.; ZIBURSKI, A. Seed dispersal in flood plain forests of Amazonia. Biotropica, v.26, v.1, p.30-43, 1994.

KUNZ, S. H. et al. Análise da similaridade florística entre florestas do Alto Rio Xingu, da Bacia Amazônica e do Planalto Central. Revista Brasileira de Botânica, v.32, n.4, p.725-736, 2009.

LIEBERMAN, D. Demography of tropical tree seedlings: a review. In: SWAINE, M. D. (Ed.). Ecology of tropical forest tree seedlings. Paris: UNESCO/Pathernon Publishing Group, 1996. p.131-138.

MAGURRAN, A. E. Ecological diversity and its measurement. London: Croom Hell Limited, 1988. 179p.

MARANGON, L. C. et al. Regeneração natural em um fragmento de Floresta Estacional Semidecidual em Viçosa, Minas Gerais. Revista Árvore, v.32, n.1, p.183-191, 2008.

MARTINS, S. V.et al. Colonization of gaps produced by death of bamboo clumps in a semideciduos mesophytic forest in south-eastern Brazil. Plant Ecology, v.172, n.1, p.121-131. 2004.

MARTINS, S. V. et al. Caracterização do dossel e do estrato de regeneração natural no subbosque e em clareiras de uma Floresta Estacional Semidecidual no município de Viçosa, MG. Revista Árvore, v.32, n.4, p.759-767, 2008.

MARTINS, S. V.; RIBEIRO, G. A. Initial secondary succession in a forest fragment disturbed by fire in Viçosa-MG, Brasil. In: VIEGAS, D. X. (Ed.). Forest Fire Research \& Wildland Fire Safety. Rotterdam: Milpress, 2002. p.1-9.

MARTINS, S. V.; RODRIGUES, R. R. Gap-phase regeneration in semideciduous mesophytic forest, south-eastern Brazil. Plant Ecology, v.163, n.1, p.51-62, 2002.

Revista Árvore, Viçosa-MG, v.38, n.1, p.31-40, 2014 
MEDLÍN, R. A.; GAONA, O. Seed dispersal by bats and birds in forest and disturbed habitats of Chiapas, México. Biotropica, v.31, n.3, p.478-485, 1999.

MEIRA-NETO, J. A. A. et al. Estrutura de uma Floresta Estacional Semidecidual Submontana em área diretamente afetada pela usina hidrelétrica de Pilar, Ponte Nova, Zona da Mata de Minas Gerais. Revista Árvore, v.21, n.3, p.338-344, 1997.

MEIRA-NETO, J. A. A.; MARTINS, F. R. Estrutura do sub-bosque herbáceo-arbustivo da Mata da Silvicultura, uma Floresta Estacional Semidecidual no município de Viçosa - MG. Revista Árvore, v.27, n.4, p.459-471, 2003.

MUELLER-DOMBOIS, D.; ELLENBERG, H. Aims and methods of vegetation ecology. New York: John Wiley \& Sons, 1974. 547p.

PAULA, A. et al. Alterações florísticas ocorridas num período de quatorze anos na vegetação arbórea de uma Floresta Estacional Semidecidual em Viçosa, MG. Revista Árvore, v.26, n.6, p.743-749, 2002.

PIELOU, E. C. Ecological diversity. New York: Jonhon Willey, 1975. 165p.

PIÑA-RODRIGUES, F. C. M.; COSTA, L. G. S.; REIS, A. Estratégias de estabelecimento de espécies arbóreas e o manejo de florestas tropicais. In: CONGRESSO FLORESTAL BRASILEIRO, 6., 1990, Campos do Jordão. Anais... Campos do Jordão: SBS/SBEF, 1990. p.676-683.

ESENDE, M. Clima e solo: suas relações com o ambiente agrícola. Informe Agropecuário, v.12, n.138, p.43-59, 1986.

REZENDE, S. B. Estudo de crono-topografia em Viçosa - Minas Gerais.. 1971. 71f. Dissertação (Mestrado em Ciência Florestal) Universidade Federal de Viçosa, Viçosa, MG, 1971.

RODRIGUES, R. R.; NAVE, A. G. Heterogeneidade florística das matas ciliares. In: RODRIGUES, R. R.; LEITÃO-FILHO, H. F. (Ed.). Matas ciliares: conservação e recuperação. São Paulo: Edusp/ FAPESP, 2000. p.45-71.

SEVILHA, C. S. et al. Fitossociologia de um trecho de Floresta Estacional Semidecidual do Jardim Botânico da Universidade Federal de Viçosa (face sudoeste), Viçosa, MG. Revista Árvore, v.25, n.4, p.431-443, 2001.

Revista Árvore, Viçosa-MG, v.38, n.1, p.31-40, 2014
SHEPHERD, G. J. Fitopac 2.1 - Campinas: Departamento de Botânica, Universidade Estadual de Campinas, 2010.

SILVA JÚNIOR, W. M. et al. Regeneração natural de espécies arbustivo-arbóreas em dois Trechos de uma Floresta Estacional Semidecidual, Viçosa, MG. Scientia Forestalis, v.66, p.11, 2004.

SOUZA, P. B. et al. Florística e estrutura da vegetação arbustivo-arbórea do sub-bosque de Eucalyptus grandis W. Hill ex Maiden em Viçosa, MG, Brasil. Revista Árvore, v.31, n.3, p.533543, 2007.

TABARELLI, M.; VILLANI, J. P.; MANTOVANI, W. Estudo comparativo da vegetação de dois trechos de floresta secundária no Núcleo Santa Virginia, Parque Estadual da Serra do Mar, SP. Revista do Instituto Florestal, v.6, n.1, p.1-11, 1994.

UHL, C. et al. Early plant succession after cutting and burning in the upper Rio Negro Region of the Amazon Basin. Journal of Ecology, v.69, n.2, p.631-649, 1981.

VALVERDE, O. O estudo regional da Zona da Mata de Minas Gerais. Revista Brasileira de Geografia, v.20, n.1, p.1-82, 1959.

van der PIJL, L. Principles of dispersal in higher plants. 3.ed. Berlin-Heidelberg/New York: Springer-Verlag, 1982. 214p.

VELOSO, H. P.; RANGEL, A. L. R.; LIMA, J. C. A. Classificação da vegetação brasileira, adaptada a um sistema universal. Rio de Janeiro: IBGE, 1991. 123p.

VIANA, V. M. Biologia e manejo de fragmentos de florestas naturais. In: CONGRESSO FLORESTAL BRASILEIRO, 6., 1990, Campos do Jordão. Anais... Campos do Jordão: SBS/SBEF, 1990. p.113-118.

VIANELLO, R. L. Meteorologia básica e aplicações. Viçosa, MG: Universidade Federal de Viçosa, 1991. 449p.

VOLPATO, M. M. L. Regeneração natural em uma floresta secundária no domínio de Mata Atlântica: uma análise fitossociológica. 1994. 123f. Dissertação (Mestrado em Ciência Florestal) - Universidade Federal de Viçosa, Viçosa, MG, 1994. 